\title{
Synaptix: A Web Platform based on Gamification Techniques for the Study of Clinical Cases
}

\author{
Omar López Chávez, Ignacio N. Márquez, Luis-Felipe Rodríguez, \\ Jorge G. Mendoza León \\ Sonora Institute of Techonology, Department of Computer and Design, Mexico \\ omarlopch@gmail.com, ignacio_nmarquez@hotmail.com, \\ luis.rodriguez@itson.edu.mx, jorge.mendoza@itson.edu.mx
}

\begin{abstract}
In this paper we present a Web platform designed to allow medical students and practitioners to study clinical cases on their own. The main objective of this proposal is to address key problems inherent in the traditional study of clinical cases by providing a tool that implements techniques and elements of gamification, simulation, and serious games. The proposed platform offers an improved learning experience through a virtual environment that provides an alternative method for training and interpretation of clinical cases for medical examinations. Medical students and practitioners can play the role of a real doctor in a simulated office. In particular, the platform allows medical students and practitioners to learn through their mistakes without hurting human beings. In addition, this platform is designed to allow users to add new clinical cases and make them available for study. The platform was validated in a local hospital by 8 medical practitioners. The participants indicated that the platform design, the tutorials included, and the ease of use factors are satisfactory.
\end{abstract}

Keywords: medical student, clinical case, gamification, simulation, serious game.

\section{Introduction}

Gamification is the process of changing a set of traditional actions to an attractive gaming experience for the user [13]. Matallaoui et al. [11] define gamification as "the use of game design elements in a context not related to the game, is an interdisciplinary tool, where users are motivated to achieve certain behavioral or psychological results". Gamification also enables the development of immersive games in virtual environments in which users are encouraged to perform desired actions. In the academic field, gamification serves as a tool to facilitate teaching and learning processes through collaborative environments [2].

Serious games (SGs) have a high impact as an instructional tool that benefits from traditional game concepts and information and communication technologies. Serious games have allowed the implementation of simulations and realistic 
virtual environments, where players can experience adventures while acquiring, practicing, and verifying knowledge. This represents a significant opportunity for 21st century educators and trainers to improve their educational tools [1].

The traditional method for studying clinical cases is through information sources such as books, articles, and automated tests. However, this method usually leads medical students to a state of saturation, stress, and anxiety given that, for example, the quantity and complexity of clinical cases to study in exam periods is high. Moreover, the feedback received from this type of information sources is limited. The study of clinical cases by medical students and practitioners also takes advantage of the monitoring of patients in hospitals. This study of clinical cases requires observation and analysis for long periods of patients with diverse conditions, diseases, signs, and symptoms [9]. However, in hospitals such as those known as "third level hospitals", only critical patients have long stays. The majority of patients are hospitalized for short periods of time, which makes it difficult the generation and access of medical students to a greater amount of knowledge that improves their learning experience and the acquisition of skills related to clinical cases that depend on hospitalized patients.

Lifshitz [8] indicates that clinical cases can not be learned through memorization or readings or through distance education strategies. In fact, the analysis of clinical cases has a very strong affective component because it implies confrontation with illness and suffering. The discussion about the limits of the teaching of clinical cases has not been solved, but this type of learning usually requires abilities for communication, physical examination, treatment, and clinical reasoning. Lifshitz [8] also proposes a well defined structure to organize and study clinical cases: 1) the approach to the patient, 2) the collection of information, 3) the analysis of the information collected, 4) clinical procedures, 5) the diagnostic decision, 6) the decision therapist, and 7) the decision Prognosis. In this context, although there is great interest in the design and application of guidelines in clinical practices for the prevention and care of diverse health situations, greater attention must be paid to its implementation and effectiveness in various practical scenarios [5].

The simulation of clinical cases involves a set of techniques that facilitate medical students and practitioners the acquisition of knowledge and skills. In particular, techniques and methods from fields such as artificial intelligence, virtual and augmented reality, and human-computer interaction have enabled the development of platforms that incorporate virtual scenarios, simulation models, and multimedia materials to simulate different real situations (e.g., for the analysis of clinical cases) [4]. Although simulation platforms do not replace the real scenarios, these allow students to learn and practice in controlled media, contributing to improve their skills and decrease the anxiety when performing an exam or procedure. This type of platform also accelerates learning and enriches the true interactions with the patients, helping medical students to avoid states of saturation experienced with traditional learning methods [9].

In this paper, we present a platform designed to allow medical students and practitioners to study clinical cases on their own. This platform represents an 
attempt to address key problems inherent in the traditional study of clinical cases by providing a tool that implements techniques and elements of gamification, simulation, and serious games. Its design takes into account the elements and strategies that according to medical students and practitioners are required to learn and practice clinical cases [10]. The paper is structured as follows. In Section 2 we discuss related work. The proposed platform and corresponding validation are presented in Section 3 and Section 4, respectively. Finally, concluding remarks and future work are discussed in Section 5.

\section{Related Work}

The website The New England Journal of Medicine presents interactive medical clinic cases designed according to the following interaction process: presentation of the case, medical history of the patient, information of the physical examination, and finally, performs a test to provide feedback and solutions of correct and incorrect answers [6]. However, although this website shows to users the percentage of the result and the studies carried out, key elements of gamification and serious games are not considered such as dashboard, unlock, and challenge. Moreover, the interaction is based on text and 2D graphics, leaving aside the implementation of $3 \mathrm{D}$ scenarios and simulations. The inclusion of additional clinical cases by users is not allowed.

Nevin et al. [12] developed the Kaizen-Internal Medicine (Kaizen-IM) software that includes elements of gamification. This tool involved a large number of residents in a medical contest that facilitated the acquisition of new knowledge in the academic period 2012-2013 in two training programs IM (internal medicine) in the USA: the residency program in Internal Medicine at the University of Alabama at Birmingham (UAB) and the University of Alabama Program at Huntsville (UAH). The data was recorded at participant level and question. The analyzes focused on the acceptance, use, and determination of the factors associated with the loss of players (attrition) and the retention of knowledge. The Kaizen-IM data provided information on modifiable factors associated with student attrition and retention of knowledge that can serve to further enhance the educational benefits of this strategy for students. This tool is an attempt to demonstrate the benefits of incorporating elements of gamification in the learning process of medical students. However, this software does not implement $3 \mathrm{D}$ simulations, but is based on strategies based on questions and answers.

Leba et al. [7] proposed an application for the training of medical students in the field of anatomy with computed tomography images, using elements of gamification, simulation, and serious games. It was designed in the context of an educational software. This proposal represents an attempt to support modern and practical methods of examination based on real cases useful for medical students and teachers. However, this application for training was not validated in a case study, the authors proposed only design guidelines. 


\section{Synaptix}

The Sypnatix platform was designed for the study of clinical cases that follow the clinical practice guide of the federal government of Mexico [3]. Synaptix implements elements and techniques of gamification, simulation, and serious games. In particular, its design is based on a previous study by Marquez et al. [10] in which data was collected from medical students and practitioners in order to 1) identify key elements and learning strategies for the study and practice of clinical cases, and 2) define how these elements and strategies should be taken into account in the design of a learning platform that incorporates elements of gamification for the study of clinical cases (see Figure 1) [10].

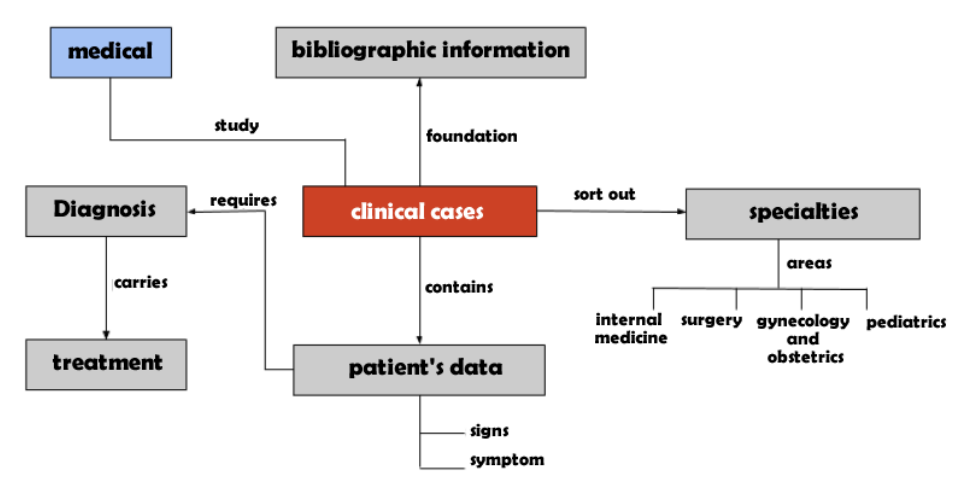

Fig. 1. Elements involved in the study of clinical cases [10].

The Synaptix platform incorporates the following elements of gamification (which are common elements reported in the literature [14]):

- Points: represent the way in which the player is observed, classified, and guided. In Synaptix, the user starts with 0 points. The points are cumulative and depend on the correct answers provided by the user.

- Badges: mark the fulfillment of goals and the constant progress of the game. Badges are activated as the user completes clinical cases on Synaptix.

- Levels: a marker for players to know where they are in a gaming experience. In Synaptix, levels are activated as progress is made in solving clinical cases.

- Dashboard: an ordered list of names and its corresponding score. Synaptix makes available information about the achievements of each user.

- Unlock: allows players to access another achievement after certain requirements are met. In Synaptix, objectives are unlocked as the user resolves clinical cases.

- Challenges: offer players an address so they know what to do within the world of the gamified experience. In Synaptix, the challenges are associated with obtaining badges. 


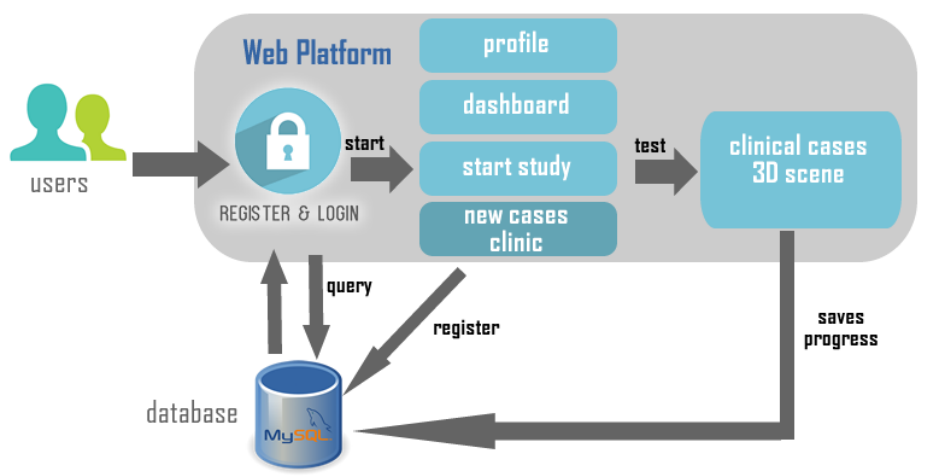

Fig. 2. The architecture of Synaptix.

Figure 2 shows the main components of the Sypnatix's architecture and their relationship: 1) the elements of gamification mentioned above, 2) a 3D virtual scenario, and 3) components related to the data management. These components create an interactive experience by enabling medical students to acquire, practice, and verify knowledge, which are key elements of SG and simulation.

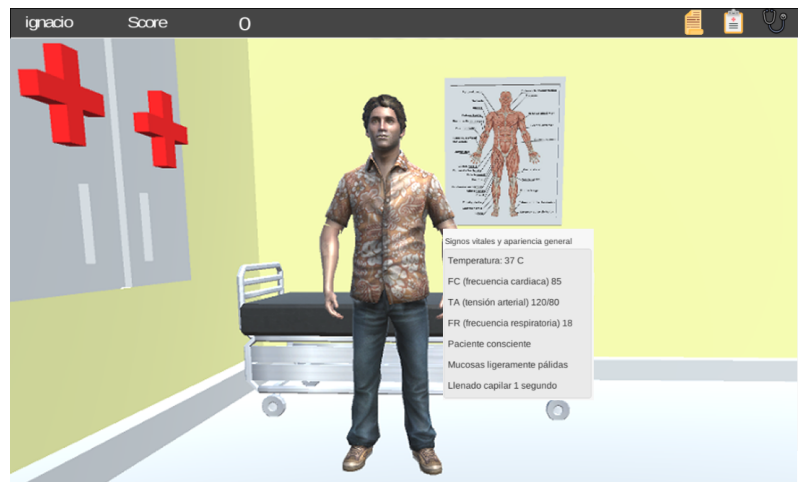

Fig. 3. Simulation of a doctor office and a virtual patient.

The virtual scenario provided by Synaptix for the study and practice clinical cases is shown in Figure 3. This virtual scenario simulates a medical office and a virtual patient. Medical students interact with the virtual patient by physically examining, for example, its lungs and head. Additional information related to clinical records or results of clinical studies (data that is sometimes taken into account in clinical cases) is also displayed in the scenario. After the medical student analyzed the data provided in the clinical case (i.e., physically examined the virtual patient and its medical records), a series of questions and possible 
answers are displayed to evaluate whether the diagnosis and treatment suggested by the student are correct. Synaptix provides feedback to medical students once an answer is submitted. If the answer is incorrect, feedback about medications or treatments is provided. The points obtained and the assigned badges are activated in the user's profile as shown in Figure 4.
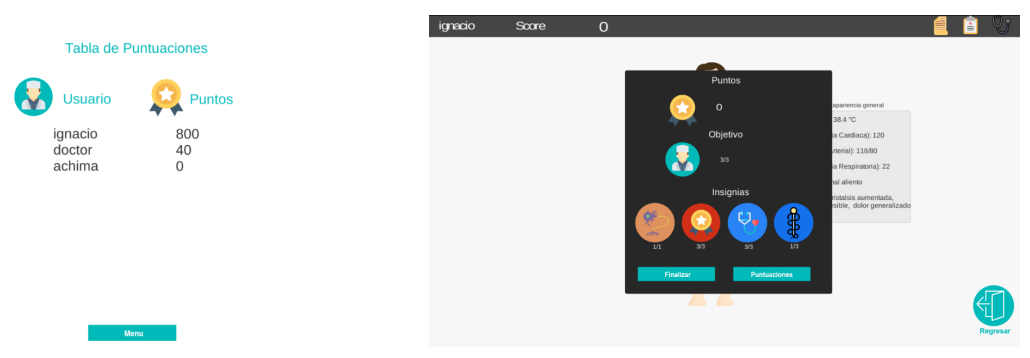

Fig. 4. Dashboards that show the scores and points obtained by medical students.

Although Synaptix is designed for the study of clinical cases related to the areas of internal medicine, surgery, gynecology and obstetrics, and pediatrics, currently only clinical cases of internal medicines are included. Nevertheless, Synaptix allows medical practitioners to include new clinical cases which are then available for their analysis by medical students. The tools used in the development of the Synaptix Web platform were Unity game development platform, Php scripting language, and the Mysql database server. In order to carry out the tests, it was posted on a web server: http://arevolution.com.mx/synaptix/

\section{Validation}

Synaptix was validated in a private local hospital by 8 medical students and practitioners ( 4 female and 4 male). The validation session consisted of an introduction by the authors about the functionality and characteristics of the platform. Afterwards, the participants used the platform to practice available clinical cases and answered a questionnaire. The instrument consisted of 29 items to measure 1) the design of the platform, 2) the instructions provided by Synaptix, and 3) easy of use factor. Figure 5 and Figure 6 show the results obtained in this evaluation phase.

The comments provided by participants include the following: 1) provide further feedback or suggest additional information sources to the user once a clinical case is carried out, 2) allow the user to make more questions to the virtual patient, 3) indicate the specific areas the medical student needs to reinforce in order to achieve better results, 4) provide greater details in the diagnosis, 5) include better medical images, and 6) include more references about the clinical cases presented. Finally, some participants emphasized the importance of badges and individual scores as incentives. 


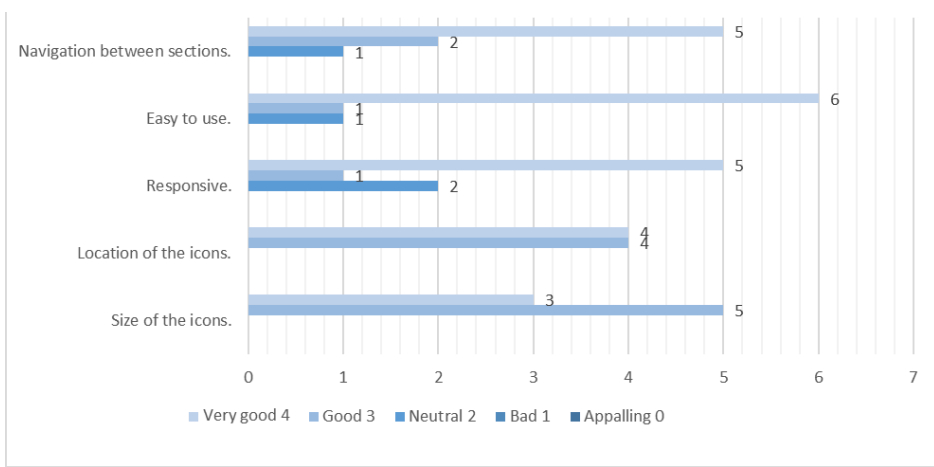

Fig. 5. Results of evaluating the design of Synaptix.

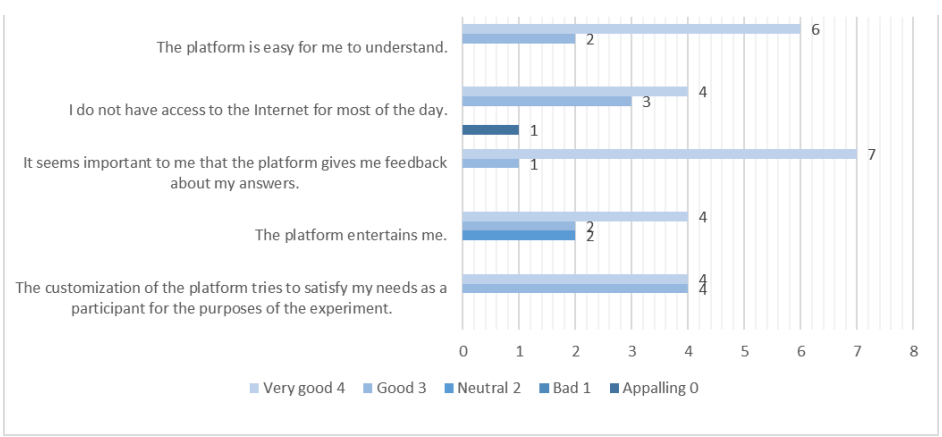

Fig. 6. Results of evaluating the easy of use of Synaptix.

\section{Conclusions and Future Work}

The main contribution of this paper is the design and implementation of a platform that 1) incorporates elements of gamification, serious games, and simulation, and 2) takes into account the elements and strategies that according to medical students and practitioners are required to learn and practice clinical cases. The Synaptix Web platform is a gamified tool that attempts to serve as an alternative method for the study of clinical cases, avoiding states of saturation, fatigue, and anxiety in medical students and practitioners. Synaptix was validated in a private local hospital by 8 medical students and practitioners. The results demonstrated that participants find that the factors associated with the design and easy of use of the platform is in general satisfactory. Future research involves an evaluation to measure complex individual's aspects such as motivation, learning, and user engagement as well as the incorporation of virtual 
reality and augmented reality components in order to create more immersive scenarios. Furthermore, AI techniques will be incorporated to enable the virtual patients to develop some human-like behaviors.

Acknowledgment. This work was supported by PFCE 2018.

\section{References}

1. Arnab, S., Lim, T., Carvalho, M.B., Bellotti, F., De Freitas, S., Louchart, S., Suttie, N., Berta, R., De Gloria, A.: Mapping learning and game mechanics for serious games analysis. British Journal of Educational Technology 46(2), 391-411 (2015)

2. Burke, B.: Gamify: How gamification motivates people to do extraordinary things. Routledge (2016)

3. Cenetec: Clinical practice guide. https://cenetec-difusion.com/gpc-sns/ (2018)

4. Chen, L., Day, T.W., Tang, W., John, N.W.: Recent developments and future challenges in medical mixed reality. In: IEEE International Symposium on Mixed and Augmented Reality (ISMAR). pp. 123-135. IEEE (2017)

5. DiCenso, A., Guyatt, G., Ciliska, D.: Evidence-Based Nursing-E-Book: A Guide to Clinical Practice. Elsevier Health Sciences (2014)

6. Group, N.: interactive medical case. https://www.nejm.org/multimedia/ interactive-medical-case (2018)

7. Leba, M., Ionica, A., Apostu, D.: Educational software based on gamification techniques for medical students. In: Proceedings of the 5th International Conference on Applied Informatics and Computer Theory (AICT). pp. 225-230 (2014)

8. Lifshitz, A.: La nueva clínica. Intersistemas Editores (2014)

9. Makransky, G., Bonde, M.T., Wulff, J.S., Wandall, J., Hood, M., Creed, P.A., Bache, I., Silahtaroglu, A., Nørremølle, A.: Simulation based virtual learning environment in medical genetics counseling: an example of bridging the gap between theory and practice in medical education. BMC medical education 16(1), 98-107 (2016)

10. Márquez, I., Mendoza, J.G., Rodríguez, L.F.: Identificación de elementos clave en el estudio de casos clínicos para su gamificación. In: Prieto, M., Pech, S., Francesa, A. (eds.) Tecnologías y Aprendizaje. Investigación y Práctica, pp. 132-139 (2018)

11. Matallaoui, A., Herzig, P., Zarnekow, R.: Model-driven serious game development integration of the gamification modeling language gaml with unity. In: 48th Hawaii International Conference on System Sciences (HICSS). pp. 643-651. IEEE (2015)

12. Nevin, C.R., Westfall, A.O., Rodriguez, J.M., Dempsey, D.M., Cherrington, A., Roy, B., Patel, M., Willig, J.H.: Gamification as a tool for enhancing graduate medical education. Postgraduate medical journal pp. 121-130 (2014)

13. Robson, K., Plangger, K., Kietzmann, J.H., McCarthy, I., Pitt, L.: Is it all a game? understanding the principles of gamification. Business Horizons 58(4), 411-420 (2015)

14. Vargas-Enríquez, J., García-Mundo, L., Genero, M., Piattini, M.: Análisis de uso de la gamificación en la enseñanza de la informática. In: Actas de las XXI Jornadas de la Enseñanza Universitaria de la Informática. pp. 105-112. Universitat Oberta La Salle (2015) 